\title{
Real Analysis in Paraconsistent Logic
}

\author{
Maarten McKubre-Jordens* Zach Weber ${ }^{\dagger}$
}

\begin{abstract}
This paper begins an analysis of the real line using an inconsistencytolerant (paraconsistent) logic. We show that basic field and compactness properties hold, by way of novel proofs that make no use of consistencyreliant inferences; some techniques from constructive analysis are used instead. While no inconsistencies are found in the algebraic operations on the real number field, prospects for other non-trivializing contradictions are left open.
\end{abstract}

This paper has two aims. The first is to show that, by weakening the logic within which we work, so as to allow for the possibility of non-trivial inconsistency, we are still able to do everyday mathematics. We see why such practice is possible: We take classical proofs, note the obstacles from a paraconsistent viewpoint, and then reconstruct the arguments, using only inferences that are valid in nontrivial inconsistent contexts. This 'breakdown' of analysis enables a fuller understanding of the fine structure of proofs. The strategy is to reverse engineer classical results (cf. [21, sec.16.1]), eventually deriving a paraconsistent basis for analysis, and this paper is a step in that direction. A fundamental result is the Heine-Borel theorem, characterising compactness of bounded closed intervals.

The second aim of the paper is to explain, in part, a basic historical fact: the original calculus of Newton and Leibniz was inconsistent - in the sense that the original definition of derivative involves a number $\varepsilon$ which is at some points non-zero (to permit division), and at other points zero (to allow 'infinitessimal' quantities to vanish); see [7, 20] 1] Yet, despite the inconsistency, early practitioners were able to draw meaningful and useful conclusions. Despite contradictions, they got the right answers. Accounting for how this is possible provides one motivation for working in a paraconsistent logic - reconstructing structures in which inconsistent reasoning actually did take place, and yet did so without falling into incoherence.

\footnotetext{
*University of Canterbury; maarten.jordens@canterbury.ac.nz

†University of Melbourne; zweber@unimelb.edu.au

${ }^{1}$ The work in this paper differs strongly from 7 , in which all the reasoning takes place in consistent contexts. There are also versions of non-trivial inconsistent calculus [17, [18, 10] (in [1]), again involving a different approach. By contrast, this paper is not a model-theoretic development, but rather axiomatic.
} 
Our interest here is in compactness properties. It turns out that, to understand the Heine-Borel theorem in a paraconsistent system, detours through ideas from constructive analysis are needed; e.g. Brouwer's fan theorem plays a key role [6, §5.3]. This is something of a surprise, since paraconsistency has often been taken as a dual or opposite to constructive, paracomplete theories [18, ch. 11], [8] It has been quipped of constructive mathematicians that they perform mathematics with one hand tied behind their back, working without universal application of the law of excluded middle. Here we tie the other hand behind our backs, and work without disjunctive syllogism or general forms of reductio. Constructive mathematicians have developed powerful tools to overcome truth value gaps; here, by restoring excluded middle, but considering the possibility of truth-value gluts, those techniques become an extremely effective way to push through proofs - a way to preserve important parts of real analysis under the possibility of inconsistency.

\section{Logic}

First we describe a paraconsistent logic. We do not make much of the logic per se, except to note its main features and to suggest that it is a good candidate for embedding paraconsistent real analysis. See the Appendix $\$ 6$ for details.

Crucially, for our logic to be inconsistency-tolerant but nontrivial, it is necessary to characterize a consequence relation $\vdash$ such that $A, \neg A \nvdash B$, for arbitrary $A, B$ - that is, the logic must be nonexplosive. (The logic will be weaker than classical logic, not validating ex contradictione quodlibet.) We also want a logic strong enough to validate inferences like modus ponens and even reductio (if $A \vdash \neg A$, then $\neg A$ ). The logic, given in the Appendix, follows the Australasian school of paraconsistent, relevant logic [26], [15, 222, and fits these needs well; it has been shown to form a solid basis for paraconsistent set theory [4], 27]. In the next sections, we only point out a few important features.

\subsection{Invalid Inferences}

An inference is invalid if it does not preserve truth, and in the context of inconsistency one must take extra care. By the definition of paraconsistency, the explosion rule $A, \neg A \vdash B$ is not allowed. But then neither is anything that validates explosion. To this end, disjunctive syllogism,

$$
A, \neg A \vee B \vdash B,
$$

\footnotetext{
${ }^{2}$ Goodman [13] saw the duality but rejected it. Still earlier, da Costa 11] had intuitions of duality between the logic $C_{1}$ and intuitionism (although there turned out not to be).
} 
is invalid in this context, due to C.I. Lewis' famous argument in [14, p. 250].

In classical logic, $\neg A \vee B$ is read as $A$ implies $B$. Since disjunctive syllogism fails, though, we cannot read $\neg A \vee B$ this way; otherwise we would be saying that modus ponens fails, too. Since modus ponens is inviolably true, we introduce a new implication connective, $\rightarrow$. A crucial point to note about this connective is that the deduction theorem is not in general true. That is, we do not have the property:

$$
\text { If } A \vdash B \text {, then } \vdash A \rightarrow B \text {. }
$$

So a deduction, marked by $\vdash$, is very different from an implication, marked by $\rightarrow$. In particular, $\rightarrow$ obeys contraposition, while $\vdash$ does not. More about this in the next section.

\section{$1.2 \quad$ Restricted Quantifiers}

In the following we make use of restricted quantifiers; cf. Brady [3, p. 327]; Butchart 9]. Restricted quantification, the formalization of the statement 'all $A$ s are $B \mathrm{~s}^{\prime}$, looks like this:

$$
\forall x \in A(x \in B)
$$

where we write $x \in A$ interchangeably with $A(x)$, thereby supposing in the background some set $A=\{z: A(z)\}$.

The key behavior of these quantifiers is to validate the following:

$$
\text { If } x \in A \vdash x \in B \text {, then } \vdash \forall x \in A(x \in B) \text {. }
$$

Concretely, in what follows we are concerned almost exclusively with the (nonempty) set $\mathbb{R}$, and want to prove theorems of the form 'all real numbers are $B$.' By the above rule, when we can prove $x \in B$ from the assumption that $x \in \mathbb{R}$, where use of other background assumptions is also allowed, then we can write the formula: $\forall x \in \mathbb{R}(x \in B)$.

Restricted quantifiers are intended to be stronger than classical material implication, because they obey modus ponens,

$$
\forall x \in A(x \in B), a \in A \vdash a \in B
$$

but weaker than our arrow-implication (so they are not simply shorter ways of saying $\forall x(A x \rightarrow B x))$, because they obey de Morgan exchanges,

$$
\begin{array}{lll}
\forall x \in A(x \in B) & \dashv & \neg \exists x \in A(x \notin B) \\
\exists x \in A(x \in B) & \dashv-\quad \neg \forall x \in A(x \notin B)
\end{array}
$$


as well as 'weakening':

$$
\forall x(x \in A), \vdash \forall x \in B(x \in A)
$$

The point is to have sentences of our language that are about what we can prove. In particular, there are often cases where we want to say that, since everything is $A$, then all $B$ s are $A$; but this statement is significantly weaker than anything expressed by $\rightarrow{ }^{3}$

Finally, in introducing these quantifiers it is essential to look out for Curry's paradox [16, 2]; see the final note of our $\S$ Appendix.

While these quantifiers are not yet widespread in the literature, we think the pages ahead makes a good test case for their being natural and useful.

\subsection{A note on absurdity}

We include the 'zero-place connective' $\perp$. This symbol is characterised by the property:

$$
\perp \vdash A,
$$

for all $A$. Throughout, when $\perp$ is derivable, it will mean that the system trivializes the real numbers. For the present purpose, it will suffice to define $\perp$ as the sentence $0=1$. This makes sense from the standpoint of von Neumann ordinals and set theoretic identity $\rfloor^{4}$ Letting $0=\emptyset=\{x: \forall y x \in y\}$ and $1=\{0\}$, then if $0=1, \forall x(x \in 1 \rightarrow x \in 0)$. Since $0 \in 1$, we would have $0 \in 0$, and then $x \in y$, for any $y$; so $x \in\{z: A\}$, where $A$ is any sentence at all. This justifies the definition and 'absurdity' property of $\perp$.

While the disjunctive syllogism is not valid in full generality, it is valid in the following sense: If $A \vee B$, and $\perp$ follows from $A$, then $B$ follows. This characterises the idea that while the system ought to tolerate inconsistencies, it should not degenerate into triviality. Employing a restricted form of disjunctive syllogism using $\perp$ is no assurance of consistency; but it does appear to be unavoidable, and is a very useful way to reason. One simple application is shown in the next section.

\footnotetext{
${ }^{3}$ Since the logic is 'relevant' it does not include weakening, $A \vdash B \rightarrow A$. With weakening, we would have to drop contraposition. Else, we could argue from $A$ to $\neg B \rightarrow A$, then to $\neg A \rightarrow B$. But if also $\neg A$ holds, i.e. $A$ is a true contradiction, then $B$ follows by modus ponens, where $B$ is arbitrary. The improper inference here is just $A \vdash \neg A \rightarrow B$, a form of explosion. Conversely, to ensure that these quantifiers do not simply amount to smuggling material implication back in requires that $\forall x(\neg A \vee B)$ does not entail $\forall x \in A(x \in B)$.

${ }^{4}$ Though see the first paragraph of $₫ 2$ below. Note too that $0=1$ can actually hold in some models of paraconsistent arithmetic, without leading to explosion [18, p. 18]. So taking $0=1$ as absurdity may not be invariant across all possible paraconsistent mathematical arrangements, especially in contexts where the symbols ' 0 ' and ' 1 ' are not defined.
} 


\subsection{Subsets and Complementation}

Restricted quantifiers and an absurdity operator now allow us to fix some notation.

Definition 1. $X \subseteq Y:=\forall z \in X(z \in Y)$ means that $X$ is a subset of $Y$, while $X \subset Y:=X \subseteq Y \wedge X \neq Y$ means that $X$ is a proper subset of $Y$. Any $x$ is in the complement of $Y$ in $X$, written $X \backslash Y$, iff both $x \in X$ and $x \in Y \vdash \perp$.

To put matters concretely, we will want to discuss

$$
x \in \mathbb{R} \backslash\{0\}
$$

which is all the $x \in \mathbb{R}$ excluding 0 . The phrasing of the definition of complement ${ }^{5}$ rules out $0 \in \mathbb{R} \backslash\{0\}$, (which otherwise would still be paraconsistently compatible with $0 \notin \mathbb{R} \backslash\{0\})$. See 2.1 below.

We similarly state the immediate consequence of definition 1 in concrete terms, although obviously $\mathbb{R}$ could be anything.

Proposition 1. If $\mathbb{R}=A \cup B$, then $\mathbb{R} \backslash A \subseteq B$.

Proof. If $x \in \mathbb{R} \backslash A$, then $x \in \mathbb{R}$ but if $x \in A$ then $\perp$. Yet if $x \in \mathbb{R}$, then by hypothesis $x \in A$ or $x \in B$. Arguing by cases, $x \in B$.

Properties like this are useful to bear in mind during left-half/right-half type arguments about intervals such as in 4 .

\section{The Real Numbers}

A paraconsistent logic allows fine distinctions where classical logic sees only equivalences ${ }^{6}$ Where any one of many arrangements and phrasings will yield the same classical theory, here the precise way definitions and axioms are stated is decisive for the success or failure of proving theorems. For instance, strict inequality $x<y$ is often glossed as $\exists z(z>0 \wedge x+z=y)$. As a definition, this would make the proof of theorem 2 much more difficult, or even impossible, because the existential quantifier gets in the way. On the other hand, this gloss on $<$ follows as theorem 13 . So each choice is much more delicate than a classical analyst might realize. This is a theme that will repeat as we go along.

\footnotetext{
${ }^{5}$ The definition of complement is a meta-clause, since it is making a conditional statement about the deducibility relation $\vdash$ of the language. The definiens is not itself a statement of the language; cf. the structural rules at \$6. This differs from a usual notion of complement $X \backslash Y:=\{x: x \in X \wedge x \notin Y\}$. This latter is meaningful, but is not enough to deliver facts like proposition 1 or its uses below, because of the invalidity of disjunctive syllogism.

${ }^{6}$ The simplest example: $A \wedge \neg A$ is distinct from $\perp$. More subtly, $x=y \wedge y=z \rightarrow x=z$ is distinct from $x=y \rightarrow(y=z \rightarrow x=z)$. C.f. $\neg \neg A$ versus $A$ in constructive settings.
} 
We assert field and order properties of a set called $\mathbb{R}$, the real numbers $]^{7}$ The positive real numbers, strictly greater than 0 , are called $\mathbb{R}^{+}$(see $\S 3$ ). The reals contain a subset $\mathbb{N}$ that is also assumed to have some standard features. The following axioms are taken as primitive, in the manner of e.g. Meyer's relevant arithmetic $R \#$, as opposed to proving that some construction based on the integers satisfies them 8

Axiom 1. The reals are a field under addition and multiplication.

Axiom 2. The reals are linearly ordered.

Axiom 3. Every nonempty set of reals bounded above has a least upper bound.

The rest of this section is devoted to spelling out in detail what it is that these axioms mean, and some implications for using them in a paraconsistent system.

\subsection{Axiom 1: The reals are a field}

Denote addition by the symbol + and multiplication by $\cdot$. Then for any $x, y, z \in$ $\mathbb{R}$, this axiom asserts the following properties:

(i) Closure: $x+y \in \mathbb{R}$, and $x \cdot y \in \mathbb{R}$;

(ii) Commutativity: $x+y=y+x$, and $x \cdot y=y \cdot x$;

(iii) Associativity: $x+(y+z)=(x+y)+z$, and $x \cdot(y \cdot z)=(x \cdot y) \cdot z$;

(iv) Existence of identity elements 0 and 1 in $\mathbb{R}: x+0=x$, and $x \cdot 1=x$;

(v) Existence of inverse elements:

the additive inverse $-x \in \mathbb{R}$, such that $x+(-x)=0$,

and the multiplicative inverse $x^{-1} \in \mathbb{R}$, such that $\left(x \cdot x^{-1}=1\right)$, which exists on the condition that $x \in \mathbb{R} \backslash\{0\}$;

(vi) Distributivity: $x \cdot(y+z)=x \cdot y+x \cdot z$.

There are some points to note here.

Firstly, in (iv), identity elements 0 and 1 are distinct, as we take $0=1$ to imply $\perp$ and thereby triviality.

\footnotetext{
${ }^{7}$ Note that this axiomatic approach is different from, but complementary to, the model theoretic approach favored by Meyer, Mortensen, et al. The latter is better for showing non-triviality; axiomatics are better, we think, for proving textbook-style theorems.

${ }^{8}$ In either [4] or [27], a development of paraconsistent set theory is provided, the latter including proofs for Peano's postulates. The set $\mathbb{N}$ exists, with many of its expected properties; the powerset of $\mathbb{N}$ exists, too. Facts about functions, mathematical induction and definitions by recursion are all covered. Such reassurance is good to have in the background; e.g. the proof of König's lemma (Lemma 19 below calls for defining a function by recursion. All the same, the arrangement here may simply be taken as axiomatic.
} 
Secondly, the condition in (v) for the existence of a multiplicative inverse to $x$ is that $x$ is any real but 0 , according to definition 1 This condition is vital: it corresponds to the usual (classical) idea that we cannot divide by 0. But the condition here must be stronger, since in a paraconsistent system it is not enough to have $x \neq 0$; we must reject the idea that $x$ could be zero; see 3.2 . theorem 12 and its consequences.

Lastly, we adopt the usual notational conventions about the symbols,$+ \cdot$, and $x^{-1}$.

\subsection{Axiom 2: The reals have a total partial order}

The set $\mathbb{R}$ is endowed with a total partial order $\leqslant$, satisfying, for each $x, y, z$ in $\mathbb{R}$ :

(i) Reflexivity: $x \leqslant x$;

(ii) Transitivity: $x \leqslant y \wedge y \leqslant z \rightarrow x \leqslant z$;

(iii) Antisymmetry: $x \leqslant y \wedge y \leqslant x \leftrightarrow x=y$;

(iv) Totality: $x \notin y \rightarrow y \leqslant x$.

(Again, see $\S$ Appendix for details on the $\rightarrow$ connective.) The properties (i) and (ii) are the requirements for $\leqslant$ to be a preorder; (iii) ensures this is a partial order; and (iv) makes this a total order. By the law of excluded middle, the totality clause can be used to derive the more usual form

$$
x \leqslant y \vee y \leqslant x,
$$

which we use liberally.

The ordering on $\mathbb{R}$ interacts with the field operations as follows. For all $x, y, z$ in $\mathbb{R}:$

(v) $x \leqslant y \vdash x+z \leqslant y+z$,

(vi) $x \leqslant y, z \geqslant 0 \vdash x z \leqslant y z$.

It is possible to do with fewer conditions here: observe that reflexivity is a consequence of totality, and that antisymmetry need not be posed as a biconditional (and so we can safely leave these conditions off, noting that $x=x$ is a consequence of reflexivity and antisymmetry of $\leqslant$, reassuring us that $=$ is indeed an equivalence relation). However, the aim here is to produce a solid, nontrivial system from which to derive properties of real numbers, and not necessarily a minimal system.

The following definition sets in place the necessary requirements for the usual strict ordering on the reals. 
Definition 2. $x<y:=x \leqslant y \wedge x \neq y$.

Theorem 2. Let $x, y, z \in \mathbb{R}$. Then:

(i) $x \nless x$

(ii) $x<y \vdash y \nless x$

(iii) $x<y, y<z \vdash x<z$

(iv) $x<y, y<x \vdash x \neq x$

(v) $x \neq y \vdash x<y \vee y<x$

(vi) $x<y, y \leqslant z \vdash x<z$

(vii) $x \leqslant y \vdash x<y \vee x=y$

(viii) $x<y \vee x=y \vee y<x$

Proof. Since $x=x$ for all real $x, \neg(x \leqslant x \wedge x \neq x)$, whence $x \nless x$, proving (i).

For (ii), if $x<y$, then $x \leqslant y$. Either $y \leqslant x$ or $y \nless x$. In the first case, since also $x \leqslant y$, this implies $x=y$, and then $y \nless x$ by definition; in the second case, $y \nless x$ by definition again.

For (iii), if $x<y$ and $y<z$, then $x \leqslant y, x \neq y, y \leqslant z$ and $y \neq z$. So $x \leqslant z$ by transitivity. Either $x=z$ or not. If not, then $x<z$ by definition. If $x=z$, then substituting $x$ for $z$ in the premises, $x=y$ by antisymmetry. Now substituting $x$ for $y$ in $y \neq z$, again $x<z$ by definition.

For (iv), if $x<y$ and $y<x$, then $x \leqslant y, y \leqslant x$, and $x \neq y$ by definition. By antisymmetry, $x=y$. Substituting, $x \neq x$.

For $(\mathrm{v})$, suppose $x \neq y$. By totality of $\leqslant$, either $x \leqslant y$ or $y \leqslant x$. In the former case $x<y$, and in the latter case $y<x$, by definition.

For (vi), from $x<y$ and $y \leqslant z$, we have $x \leqslant z$. If $x \neq z$ then we stop; if $x=z$ then by substitution $z<y$, and then $z=y$; and then $x<z$ by substitution again.

For (vii), suppose $x \leqslant y$. Either $x \neq y$ or $x=y$. In the former case, $x<y$ by definition.

Trichotomy (viii) follows from the axiom $x \nless y \rightarrow y \leqslant x$ : since $x \leqslant y \vee x \nless y$, $x \leqslant y \vee y \leqslant x$. Distributing this over $x=y \vee x \neq y$ gives the result.

\subsection{Axiom 3: The least-upper-bound principle}

Definition 3. A real number $y \in \mathbb{R}$ is an upper bound for $S \subseteq \mathbb{R}$ iff

$$
\forall x \in S(x \leqslant y) .
$$


A set of reals is bounded above if it has an upper bound. The least upper bound of $S$, called $\sup S$ (the supremum of $S$ ), is an upper bound of $S$ such that it is less than or equal to every upper bound of $S$ :

$$
\forall z \in \mathbb{R}(\forall x \in S(x \leqslant z) \rightarrow \sup S \leqslant z)
$$

The terms lower bound, bounded below, and greatest lower bound (the infimum of $S$, inf $S$ ) are defined similarly, in the obvious way; a set $S \subseteq \mathbb{R}$ is bounded if it is both bounded above and bounded below.

The following proposition makes it clear that use of the definite article is justified when referring to the least upper bound of a set of reals.

Proposition 3. The supremum of a set of reals $S$ that is bounded above is unique.

Proof. Let $y$ be a least upper bound of $S$. Then for any $x \in S, x \leqslant \sup S$ and $x \leqslant y$. Being 'least' means that, for all $z \in \mathbb{R}$,

$$
\forall x \in S(x \leqslant z) \rightarrow \sup S \leqslant z,
$$

and also that

$$
\forall x \in S(x \leqslant z) \rightarrow y \leqslant z .
$$

Since both $\sup S$ and $y$ are in $\mathbb{R}, \sup S \leqslant y$, and $y \leqslant \sup S$. Thus $y=\sup S$ by antisymmetry, as required.

Proposition 4. Let $S \subseteq \mathbb{R}$ be non-empty and bounded above. For each $y \in \mathbb{R}$, if $y$ is not an upper bound for $S$, then $\exists x \in S(y<x)$.

Proof. Suppose that $y$ is not an upper bound for $S$. Then

$$
\neg \forall x \in S(x \leqslant y)
$$

by definition 3 . By definition of $\neg \forall$, there exists $x \in S$ with $x \nless z y$. Antisymmetry contraposed gives $x \neq y$, and totality shows that $y \leqslant x$. Thus $y<x$ by definition.

This proposition establishes unusually strong linearity on $\mathbb{R}$ for a system not using disjunctive syllogism. 


\section{The Field $\mathbb{R}$}

In this section we take some notes on the algebraic aspects of the reals, in preparation for topological inquiries. We set

$$
\mathbb{R}^{+}=\{x \in \mathbb{R}: 0 \leqslant x\} \backslash\{0\}
$$

calling attention to the definition of $X \backslash\{Y\}$ in $\$ 2.1$. This is to allow division by $x \in \mathbb{R}^{+}$, about which more in $\$ 3.2$.

\subsection{Archimedean Properties}

We suppose the order $\leqslant$ on $\mathbb{N} \subseteq \mathbb{R}$ to include the following properties 9 for all $n \in \mathbb{N}$ :

(i) $n+1 \in \mathbb{N}$,

(ii) $n \leqslant n+1$,

(iii) $0<1 / n$

The next theorem uses these properties; it contains the germ of the idea we will need to prove the Heine-Borel theorem.

Theorem 5. $\mathbb{N}$ is not bounded from above in $\mathbb{R}$.

Proof. Either $\mathbb{N}$ is bounded above in $\mathbb{R}$ or it is not. If not, we are done. If there is a real upper bound for $\mathbb{N}$, then there is a least (axiom 3 ), $k=\sup \mathbb{N}$, such that

$$
\forall n \in \mathbb{N}(n \leqslant k)
$$

Since $\mathbb{N}$ is closed under addition, similarly $n+1 \leqslant k$ for all natural numbers $n$. But then $n \leqslant k-1$ for all $n \in \mathbb{N}$, and $k-1$ is also an upper bound for $\mathbb{N}$. Since $k \in \mathbb{R}$ is the least upper bound, by definition 3 , $k \leqslant k-1$. By the ordering and field properties of $\mathbb{R}, k-1 \leqslant k$. Now by antisymmetry we have $k=k-1$, which yields $0=1$ and thus $\perp$. And $\perp$ implies that $\mathbb{N}$ is unbounded above. Therefore in either case $\mathbb{N}$ the result follows.

Proposition 6. $\forall x \in \mathbb{R}^{+} \exists n \in \mathbb{N}(0<1 / n<x)$.

Proof. First note that $1 / n>0$ for all $n \in \mathbb{N}$. Now suppose

$$
\exists x \in \mathbb{R}^{+} \forall n \in \mathbb{N}(1 / n \nless x)
$$

\footnotetext{
${ }^{9}$ In [27, $\left.\S 7\right]$, the set theoretic ordinal $\omega$ and its membership/inclusion relations are shown to be appropriate representations of the natural order on $\mathbb{N}$.
} 
Then $1 / n \notin x$, and so by total order, $x \leqslant 1 / n$. Then $n \leqslant 1 / x$ for all $n \in \mathbb{N}$. This means that $1 / x$ is an upper bound for $\mathbb{N}$, and the reasoning from theorem 5 completes the proof.

Proposition 7 (Archimedes' Axiom). $\forall x \in \mathbb{R}^{+} \exists n \in \mathbb{N}(x<n)$.

Proof. If there exists $x \in \mathbb{R}^{+}$such that $x \nless n$ for all natural numbers $n$, then by definition of $<$ and totality, we get $x \geqslant n$ for all $n \in \mathbb{N}$. The reasoning from theorem 5 again completes the proof.

Corollary 8. $\forall x, y \in \mathbb{R}^{+} \exists n \in \mathbb{N}(y<x n)$.

Proof. Since $y / x \in \mathbb{R}^{+}$, by proposition 7 we have $y / x<n$, for some $n \in \mathbb{N}$. Multiply both sides by $x$.

Theorem 9 (Density of $\mathbb{R}$ ). For all $x, y \in \mathbb{R}$ with $x<y$, there exists $z \in \mathbb{R}$ such that $x<z<y$.

Proof. Assume without loss of generality that $y>0$. Since $y-x \in \mathbb{R}$, from proposition 6, we obtain $n \in \mathbb{N}$ such that $1 / n<y-x$. Then $x+1 / n<y$, while $x<x+1 / n$. Then $x+1 / n$ is the number we need.

It is convenient for later, if a bit miscellaneous, to include the following here:

Definition 4. The absolute value of any $x \in \mathbb{R}$ is given by the function ${ }^{10}$

$$
|x|= \begin{cases}x & \text { if } 0 \leqslant x \\ -x & \text { if } x<0\end{cases}
$$

Lemma 10. $x \leqslant 0 \vdash 0 \leqslant-x$.

Proof. By the interaction between order and field, if $x \leqslant 0$ then $x+-x \leqslant 0+-x$. Since $x+-x=0$ and $0+-x=-x+0=-x$, substituting gives the result.

Theorem 11 (Triangle Inequality). $|x+y| \leqslant|x|+|y|$.

Proof. If both $x, y$ are greater than or equal to 0 , then $x=|x|$ and $y=|y|$, and $|x+y|=x+y=|x|+|y|$, as needed. Similarly, if both $x, y \leqslant 0$, then $x+y \leqslant 0$, and so

$$
|x+y|=-(x+y)=-x+(-y)=|x|+|y| \text {. }
$$

Now without loss of generality suppose $x \geqslant 0$ and $y \leqslant 0$. Then $|x|+|y|=x-y$. Since $y \leqslant 0$, we have too that $-y \geqslant 0$. But $y \leqslant-y$ allows us to deduce

\footnotetext{
${ }^{10}$ The notion of a function is grounded in the standard set theoretic definitions [27, and associated function symbols are used liberally.
} 
$x+y \leqslant x-y$ by lemma 10 . Similarly, since $-x \leqslant x,-x-y \leqslant x-y$. It follows that

$$
|x+y| \leqslant x-y=|x|+|y| .
$$

as required.

\subsection{Inconsistency?}

This paper is motivated in part by considering inconsistency in the early calculus. It is now time to investigate the role of inconsistency here.

A fact first noticed by Dunn, and then reported in the paraconsistent analysis literature, e.g. [19], concerns 'dividing by zero'. For all $a$, we know $a-a=0$. But suppose $a \neq a$. Then it would appear that $a-a \neq 0$. (According to theorem 12 below, $a \neq a$ implies $a<a$, which further implies $0<a-a$.) If being non-identical with 0 were all it took to have an inverse, then we also have the existence of an $\frac{1}{a-a}$, such that

$$
\frac{a-a}{a-a}=1
$$

Because $a-a=0$, by substitution we have

$$
1=\frac{a-a}{a-a}=\frac{0}{a-a}=0
$$

which is $1=0$.

This explains our strong condition for the existence of multiplicative inverses. The number $a-a$ will not have an inverse, even if $a \neq a$. Nonetheless, even with such precautions the real line is still not entirely safe for inconsistent numbers, as we now show.

Theorem 12. The following statements are equivalent:
(i) $x \nless x$,
(ii) $x<x$,
(iii) $x \neq x$.

Proof. If (i) holds, then since $x=x$ for all $x$, (ii) follows by definition. And if (ii) holds, then again by definition $x \leqslant x \wedge x \neq x$, and therefore (iii) follows. Last, if (iii) holds, then by antisymmetry contraposed and reduction, (i) follows. Thus all three statements are equivalent.

Here follows the usual gloss on the strict ordering:

Lemma 13. $x<y \vdash \exists r \in \mathbb{R}(r>0 \wedge x+r=y)$. 
Proof. Since $x<y$ implies that $0<y-x$, and since $r=y-x>0$, using commutativity of addition and properties of the additive inverse,

$$
x+r=x+(y-x)=y+(x-x)=y .
$$

This is enough for the immediate corollary:

Corollary 14. For each $x \in \mathbb{R}$, if $x<x$ then $0<0$.

Proof. If $x<x$ then some $r>0$ is such that $x+r=x$ (lemma 13). Canceling, $r=0$. But also $r>0$, so by substitution, $0<0$ (and, by theorem $12,0 \neq 0$ ).

But this is just a particular instance of a more general theorem:

Theorem 15. Inconsistency at the level of order or identity spreads; that is,

$$
\exists x \in \mathbb{R} x<x \vdash \forall x \in \mathbb{R} x<x,
$$

and equivalently for $x \neq x, x \notin x$, as in theorem 12.

Proof. If $x<x$, then $x+y<x+y$. Then $-x+(x+y)<-x+(x+y)$, hence $(x-x)+y<(x-x)+y$, hence $y<y$ for any $y$. By theorem 12 , the same goes for $\neq$ and $\not$.

The algebra of fields is designed to preserve the relations $=$ and $\leqslant$ (and derivatively, $<$ ) under the operations of addition and multiplication. We see that such preservation is very robust here. Any inconsistency at the level of a particular single number, say some proposed infinitesimal $\varepsilon<\varepsilon$, will carry out to all the numbers $x<x$. There does not appear to be a way to have inconsistent numbers in an algebraic field, if that field is to support cancellation laws like $x+z=y+z \vdash x=y$; cf. [18, p. 43]. A fortiori any $\varepsilon=0$ for which $\varepsilon \neq=0$ will explode.

On the other hand, we emphasize that the existence of inconsistent numbers $x<x$ does not appear to follow from our axioms (all of which are rephrased classical axioms). And while we have shown that inconsistency spreads, this appears to be true only at the level of negated algebraic relations. That is, even in the event of $\forall x x<x$, the proposed axioms do not seem to lead to $\perp$. (Given our arrangement, with key principles formalized by non-contraposable rules, why would $0=1$ follow from $1 \neq 1$ ?) Such a theory would not be (logically) trivial. The theory would be very 'noisy,' so to speak, but there wouldn't necessarily be interference in deriving many commonplace results. Enough precautions are installed that the possibility of this being (a step towards) the inconsistent but non-trivial Newton-Leibniz calculus is not ruled out. 


\section{Compactness}

The purpose of this section is to prove a cornerstone of many important theorems in real analysis: the Heine-Borel compactness theorem. In order to establish it paraconsistently, once again we are very careful about how terms are defined, and the way theorems are phrased, e.g. definition 3 for theorem 18 . The sequence of arguments and strategies building up to the Heine-Borel theorem is novel; to begin this section, we outline the ideas.

\subsection{Method: Direct reasoning, finite and infinite}

The Heine-Borel theorem concerns a relationship between finite and infinite sets (via definitions 5 and 8 below). The standard argument follows a backand-forth halving strategy: suppose the relationship fails, argue down a nest of intervals (definition 6 below) by choosing each time the side of the interval for which it fails, reach a point where it holds, and thus the supposition is 'reduced to absurdity'. That will not work here, because we cannot isolate specific suppositions to reject. Given an argument from

$$
A_{0}, \ldots, A_{k}
$$

to $B$, then, supposing $B$ is a contradiction, this leaves us with only

$$
\neg A_{0} \vee \ldots \vee \neg A_{k}
$$

and without disjunctive syllogism, the argument now stops. Unless all these disjuncts reduce to absurdity, as opposed to merely being contradictory, there is no way to make the logic insist on one disjunct or another. And the backand-forth strategy employs more than one assumption to move forward.

To solve this problem, we directly examine the structure of the line, without making suppositions we plan to reject, and keeping careful track of the process of inspection. To make this precise we need a metaphor for inspecting a nest of intervals. This is where ideas from constructive analysis come in, and in particular the concept of a finite binary tree (definition 7).

Further, we must draw heavily on the logical relationship between finite and infinite, using the key lemma 17 below. This is a powerful generalization of theorem 5 , showing that the structure of $\mathbb{N}$ enforces a very sharp divide between finite and infinite. Now, though, instead of considering extrinsic bounds on $\mathbb{N}$ as they come from the least upper bound property of $\mathbb{R}$, we consider intrinsic bounds on finitude. Direct arguments are used. 
Definition 5. Let $|X|$ be the number of elements in a set $X$, its cardinality ${ }^{11}$ Then $X$ is finite iff

$$
\exists n \in \mathbb{N}(|X| \leqslant n),
$$

and $X$ is infinite iff $\forall n \in \mathbb{N} n<|X|$.

Lemma 16. Any $X$ is either finite or infinite. A fortiori, if $X$ is not infinite, it is finite.

Proof. We prove the stronger conditional claim, from which the first disjunctive claim follows by structural rules. If $X$ is not infinite, then $\exists n \in \mathbb{N}(n \nless|X|)$. Then by total order and definitions, $\exists n \in \mathbb{N}(|X| \leqslant n \vee|X|=n)$; but by antisymmetry, $|X|=n$ weakens to $|X| \leqslant n$; therefore $\exists n \in \mathbb{N}(|X| \leqslant n)$, showing finitude.

Lemma 17. If any $X$ is both finite and infinite, then $\perp$.

Proof. Let $X$ be both finite and infinite:

$$
\exists n \in \mathbb{N}(|X| \leqslant n) \text { and } \forall n \in \mathbb{N}(n<|X|) .
$$

Since $|X|>n$ for all $n \in \mathbb{N}$, also $|X| \geqslant n$ for all $n$. Since $|X| \leqslant n$ for some $n \in \mathbb{N}$, then by antisymmetry, $|X|=n$, for some $n \in \mathbb{N}$. But, by infinitude of $X$ and the ordering on $\mathbb{N}$, we have $|X|>n+1$. Therefore, $n>n+1$ by substitution, so that $n \geqslant n+1$ by definition. Since also $n \leqslant n+1$ by the ordering of the natural numbers, antisymmetry gives $n=n+1$. Subtracting $n$ from both sides entails $0=1$, which is $\perp$.

\subsection{Nested Intervals}

A sequence of real numbers is, as usual, a set of ordered pairs $\left\langle n, x_{n}\right\rangle$, i.e. a function from the natural numbers $\mathbb{N}$ to the reals $\mathbb{R}$; we call $x_{n}$ the $n^{\text {th }}$ term of the sequence. Often we denote a sequence by $\left(x_{n}\right)_{n=0}^{\infty},\left(x_{n}\right)_{n \geqslant 0}$, or simply $\left(x_{n}\right)$.

The basic topological concept is open set, and open subset 12

Definition 6. A subset $S$ of $\mathbb{R}$ is open if

$$
\forall x \in S \exists r \in \mathbb{R}^{+}(|x-y|<r \rightarrow y \in S)
$$

A subset $S$ of $\mathbb{R}$ is closed iff its $\mathbb{R} \backslash S$ is open.

\footnotetext{
${ }^{11}$ Not to be confused with the notation for absolute values. For a specification of cardinality in a paraconsistent set theory, see 4 p. 315]. No special properties of a theory of cardinal numbers are needed here, save that such a theory exists, and that the $\leqslant$ we have been studying is the same $\leqslant$ that orders the transfinite cardinals, but restricted to $\mathbb{R}$.

${ }^{12} \mathrm{It}$ is worth pointing out that the logic of open sets has been said to be naturally intuitionistic, and that of closed sets naturally paraconsistent [18 p.103].
} 
$A$ closed interval from $a$ to $b$ is the set $[a, b]=\{x: a \leqslant x \wedge x \leqslant b\}$. A nest of intervals is a sequence of intervals $\left(\left[a_{n}, b_{n}\right]\right)_{n=0}^{\infty}$ such that

$$
\left[a_{n+1}, b_{n+1}\right] \subseteq\left[a_{n}, b_{n}\right]
$$

for all $n$. A decreasing nest is a nest such that $\left[a_{n}, b_{n}\right] \subset\left[a_{n+1}, b_{n+1}\right]$.

If $I=[a, b]$ is a closed interval of real numbers, then $[a,(a+b) / 2]$ is the closed left-half of $I$, and $[(a+b) / 2, b]$ is the closed right-half of $I$.

Theorem 18 (Nested Intervals Theorem). The intersection of a nested sequence of closed intervals in $\mathbb{R}$ is nonempty; that is, given a nest of intervals $\left(\left[a_{n}, b_{n}\right]\right)_{n=0}^{\infty}$, there exists $x \in \mathbb{R}$ such that $x \in\left[a_{n}, b_{n}\right]$ for all $n$.

Proof. Suppose $\left(\left[a_{n}, b_{n}\right]\right)_{n=0}^{\infty}$ is a nest of intervals. Since the sequence $\left(a_{n}\right)$ is bounded above by any member of $\left(b_{n}\right), \sup a_{n}=\xi$ exists; by definition $3, \xi \leqslant b_{n}$ for all $n \in \mathbb{N}$. So for each $n, a_{n} \leqslant \xi \leqslant b_{n}$. That is, $\xi \in\left[a_{n}, b_{n}\right]$ for each $n$, whence

$$
\xi \in \bigcap_{n=0}^{\infty}\left[a_{n}, b_{n}\right]
$$

as required.

\subsection{Trees}

Definition 7. $A$ tree is a partially ordered set $\langle T, \preccurlyeq\rangle$ such that for every $\alpha \in T$, the set $\{\beta \in T: \beta \preccurlyeq \alpha\}$ is linearly ordered by $\preccurlyeq$. The elements of $T$ are called vertices. A linearly ordered subset of $T$ is called a branch or a path through $T$.

$A$ vertex $\alpha^{\prime} \in T$ is an immediate successor of $\alpha \in T$ iff

$$
\alpha \preccurlyeq \alpha^{\prime} \wedge \forall \beta \in\left\{x: \alpha \preccurlyeq x \preccurlyeq \alpha^{\prime}\right\}\left(\beta=\alpha \vee \beta=\alpha^{\prime}\right) .
$$

$A$ binary tree is a tree in which every $\alpha \in T$ has at most two immediate successors $\alpha^{\prime}, \alpha^{\prime \prime}$ in the partial order.

A tree is finite if the set $T$ is. A path $p \subseteq T$ has length $|p|$, and the number of vertices below a vertex $\alpha$ is $|\alpha|$.

Lemma 19 (König's Lemma). Let $T$ be a binary tree. If $T$ is infinite, then $T$ has an infinite path.

Proof. Let $T$ be an infinite binary tree, and let $\alpha_{0}$ be the first (topmost) vertex of $T$. We inductively construct an infinite path throught $T$ as follows.

Let $\alpha_{n}$ be a vertex. Since $T$ is a binary tree, $\alpha_{n}$ has at most two vertices directly below it, $\alpha^{\prime}$ and $\alpha^{\prime \prime}$. If both $\left|\alpha^{\prime}\right|$ and $\left|\alpha^{\prime \prime}\right|$ are finite, then $\left|\alpha_{n}\right|$ is also 
finite, because

$$
\left|\alpha_{n}\right| \leqslant\left|\alpha^{\prime}\right|+\left|\alpha^{\prime \prime}\right|+2 .
$$

Now suppose $\alpha_{n}$ has infinitely many vertices below it (as is certainly true of $\alpha_{0}$, since $T$ is infinite). By excluding the middle, either at least one of $\left|\alpha^{\prime}\right|,\left|\alpha^{\prime \prime}\right|$ is infinite, or both $\left|\alpha^{\prime}\right|$ and $\left|\alpha^{\prime \prime}\right|$ are finite. In the latter case, $\left|\alpha_{n}\right|$ is both finite, by (1), and infinite, by assumption. Then by lemma 17, at least one of $\left|\alpha^{\prime}\right|,\left|\alpha^{\prime \prime}\right|$ is infinite. Therefore there is an immediate successor with infinitely many vertices below it.

So for each $n$, choose $\alpha_{n+1}$ a vertex directly below $\alpha_{n}$ with infinitely many below. Formally, define by recursion

$$
\begin{aligned}
\alpha_{0} & =\alpha_{0} \\
\alpha_{n+1} & = \begin{cases}\alpha^{\prime} & \text { if }\left|\alpha^{\prime}\right| \text { is infinite } \\
\alpha^{\prime \prime} & \text { otherwise }\end{cases}
\end{aligned}
$$

where $\alpha^{\prime}, \alpha^{\prime \prime}$ are the immediate successors of $\alpha_{n}$. This gives an infinite path $\alpha_{0} \alpha_{1} \alpha_{2} \ldots$ through $T$.

It is the classical contrapositive of König's Lemma that we need. A direct proof is again provided.

Theorem 20 (Brouwer's Fan Theorem). Let $T$ be a binary tree. If every path through $T$ is finite, then $T$ is finite.

Proof. Either $T$ is finite or it is infinite. Suppose $T$ is infinite. Then, by König's Lemma, there exists an infinite path $p$ through $T$. Since every path through $T$ is finite, $|p|$ is finite; so $|p|$ is both finite and infinite. By lemma 17 it follows that $T$ is finite. Thus, in either case, $T$ is finite.

Brouwer's Fan Theorem is a fundamental theorem of Brouwer's intuitionism (though not of all varieties of constructive mathematics, for there are recursive counterexamples; see [6, Ch. 3]). Its use here is striking, since paraconsistent logic can be thought of as dual to intuitionism [18, ch. 11], [8. It is precisely the similarity of the fan theorem - and the non-acceptability of the latter in some forms of constructivism - to notions of compactness that blocks constructive proofs for theorems such as Heine-Borel.

\subsection{The Heine-Borel Theorem}

One last preliminary observation is needed. It hints at intrinsic interest worthy of further study, which we leave for now to future work on paraconsistent topol- 
ogy. Note that both the closed left-half and the closed right-half of a closed interval of real numbers are closed. This follows immediately from definition 6 .

Lemma 21. Any closed interval of real numbers is equal to the union of its closed halves.

Proof. By trichotomy (proposition 2), it follows that if $x$ is any real number in a closed interval $I$, then $x$ is either in the closed left-half or the closed right-half of $I$ (or both). If $x$ is in either the closed left-half or closed right-half interval, then since each closed half is a subset of $I, x$ is in $I$. Thus $I$ is the union of its closed halves.

Definition 8. A collection of sets $\mathcal{Y}$ is a cover of $X$ iff for every $x \in X$, there is a $Y \in \mathcal{Y}$ such that $x \in Y$. Any $\mathcal{Z}$ is a subcover of $X$ when $\mathcal{Z} \subseteq \mathcal{Y}$ and $\mathcal{Z}$ is a cover of $X$. An interval is $I$ compact if every open cover of $I$ contains a finite subcover of $I$.

Theorem 22 (Heine-Borel). Every bounded closed interval of $\mathbb{R}$ is compact.

Proof. Let $\mathcal{U}$ be an open cover of a bounded closed interval $I$. Let $I_{1}$ be a closed half of $I$, let $I_{2}$ be a closed half of $I_{1}$, and so forth. By this procedure, we construct a nested sequence of closed intervals $I \supset I_{1} \supset I_{2} \supset \ldots$ such that, for each $n$,

$$
\left|I_{n}\right|=2^{-n}|I|
$$

The nested intervals principle (theorem 18) now gives us some

$$
\xi \in \bigcap_{n=1}^{\infty} I_{n} .
$$

Since $\mathcal{U}$ is an open cover, there exists an open set $U \in \mathcal{U}$ such that $\xi \in U$. By the definition of open set, there is some $r>0$ such that $|x-\xi|<r$ implies $x \in U$. Now choose $N \in \mathbb{N}$ such that $2^{-N}|I|<r$. Then for each $x \in I_{N}$,

$$
|x-\xi| \leqslant 2^{-N}|I|<r
$$

which implies that $x \in U$. So $I_{N} \subseteq U$ and hence for each $n \geqslant N, I_{n}$ admits a finite subcover - namely, the open set $U$. Thus, any sequence of closed halfintervals eventually admits a single open cover.

Consider the collection $T_{I}$ of sequences formed by taking the collection of all sequences of closed half-intervals and truncating each sequence after the first member where it admits a single open cover 13 The result corresponds to a

\footnotetext{
${ }^{13}$ The meaning of phrases like 'truncating after the first members such that...' can be made perfectly precise, as per definition 3
} 
binary tree for which each path is finite. Theorem 20 now ensures that this tree is finite.

It remains to identify a collection that is a finite subcover of the interval. Consider the set $\mathcal{V}$ of all $U \in \mathcal{U}$ such that $U$ covers the last member of some path through $T_{I}$. First, $\mathcal{V} \subseteq \mathcal{U}$. Second, for every $x \in I$, it follows from lemma 21 that $x$ belongs to one of the $I_{n}$ contained in some $U \in \mathcal{V}$. Finally, since $T_{I}$ is finite, $\mathcal{V}$ is finite, as was to be shown.

\section{Conclusion}

The paraconsistent approach promises to recover an essential core of classical analysis. With a few more familiar notions on convergence, we could shed more light on the the real line - showing the completeness of $\mathbb{R}$. Further, the Bolzano-Weierstrass theorem states that every bounded, infinite sequence in $\mathbb{R}$ has a convergent subsequence; the proof of this theorem is another variation on the nested intervals property and associated arguments (theorem 18), requiring only an additional squeezing lemma $\sqrt{14}$ But here is not the place to do justice to these ideas. Enough has been put forward to show that, with suitable and intuitive background assumptions, a paraconsistent development of analysis is not only viable, but it is rich.

What can the reader, who may be a classical mathematician, take out of the results so far obtained? We cannot claim that the proofs are constructive; some are not. Yet the proofs do seem to offer kinds of information that classical arguments do not. One such feature that our arrangement makes clear is: what can and cannot be inconsistent. For example, only two very distinctive points of consistency-on-pain-of-absurdity (at the finite/infinite boundary (lemma 17), and at $0=1$ ) have been sufficient to derive compactness results. Meanwhile, the possibility of a natural inconsistency arising at some other level is prepared for, since we have used only paraconsistently valid deductions ${ }^{15}$ These are first steps to understanding the full, unreconstructed ideas of calculus and topology.

More expansively, and in keeping with one of the original motivations for the particular logic used here, we suggest that the inferences in the proofs are more relevant than what is proved using classical ex contradictione quodlibet. If we cannot go from $A \wedge \neg A$ to $B$ without some discerning reason, then inconsistent assumptions concerning $A$ will not shed any light on $B$, whether those assumptions are true or not. Allowing inconsistent counterexamples carves the details of reasoning, of proof and structure, more finely than the classical or constructive analysis. The typical classical proof can obscure content of the

\footnotetext{
${ }^{14}$ For functions $f<g<h$, if $f$ and $g$ go to the same limit, then $g$ does, too.

${ }^{15}$ Noting again that neither inconsistency nor non-triviality have yet been demonstrated.
} 
theorem by a reductio; this is well-known from the perspective of constructive logics, but has been less appreciated in other logics. Now, bridging the gap between non-classical mathematics, combining techniques, has begun. In doing so, even more made visible - only without the assumption of consistency.

\section{Acknowledgements}

Thanks to Douglas Bridges, Chris Mortensen, Graham Priest, Greg Restall, and the audience at the 2010 Australasian Association of Logic conference, for help with this paper.

\section{Appendix: Logic}

The following is a Hilbert-style presentation of a relevant, paraconsistent logic called $D K Q$; see [24, [5] (in 23]), [4], and the note on Curry below. The language is that of first order logic with identity. The usual shorthand is used: $A \vee B$ for $\neg(\neg A \wedge \neg B) ; A \leftrightarrow B$ for $(A \rightarrow B) \wedge(B \rightarrow A) ; \exists$ is $\neg \forall \neg$.

\section{Axioms}

All instances of the following schemata are theorems:

$$
\begin{array}{lll}
I & A \rightarrow A & \\
\text { IIa } & A \wedge B \rightarrow A & \\
\text { IIb } & A \wedge B \rightarrow B & \\
\text { III } & A \wedge(B \vee C) \rightarrow(A \wedge B) \vee(A \wedge C) & \text { (distribution) } \\
I V & (A \rightarrow B) \wedge(B \rightarrow C) \rightarrow(A \rightarrow C) & \text { (conjunctive syllogism) } \\
V & (A \rightarrow B) \wedge(A \rightarrow C) \rightarrow(A \rightarrow B \wedge C) & \\
V I & (A \rightarrow \neg B) \rightarrow(B \rightarrow \neg A) & \text { (contraposition) } \\
V I I & \neg \neg B \rightarrow B & \text { (double negation elimination) } \\
V I I I & A \vee \neg A & \text { (excluded middle) } \\
\text { IX } & (\forall x) A \rightarrow A(a / x) & \\
X & (\forall x)(A \rightarrow B) \rightarrow(A \rightarrow(\forall x) B) & \\
X I & (\forall x)(A \vee B) \rightarrow A \vee(\forall x) B & \\
& &
\end{array}
$$

Axioms $X$ and $X I$ have the caveat that $x$ does not appear free in $A$. Note that the law of excluded middle implies, by definition of connectives, the law of non-contradiction, $\neg(A \wedge \neg A)$. This does not preclude contradictions, though; if there are any $B$ such that $B \wedge \neg B$, then the law of non-contradiction is itself contradictory, too. Excluded middle also makes the following rule valid:

$$
A \rightarrow B \vdash \neg A \vee B
$$


The converse fails. This shows in a precise way how our arrow is stronger than standard material implication.

\section{Rules}

The following rules are valid:

$$
\begin{array}{lll}
I & A, B \vdash A \wedge B & \text { (adjunction) } \\
\text { II } & A, A \rightarrow B \vdash B & \text { (modus ponens) } \\
\text { III } & A \vdash(\forall x) A & \text { (universal generalization) } \\
I V & A \rightarrow B, C \rightarrow D \vdash(B \rightarrow C) \rightarrow(A \rightarrow D) & \text { (hypothetical syllogism) } \\
V & x=y \dashv A(x) \leftrightarrow A(y) & \text { (substitution) }
\end{array}
$$

Rule $V$ characterizes $=$ as an equivalence relation.

\section{Structural rules}

$$
\frac{A \vdash B}{A \vee C \vdash B \vee C} \quad \frac{A \vdash B}{\exists x A \vdash \exists x B}
$$

These (called meta-rules by Meyer and the Brady) validate the argument:

$$
\text { if } A \vdash B \text {, and } \neg A \vdash B \text {, then } A \vee \neg A \vdash B \text {. }
$$

Since excluded middle is a property of our logic, this is sufficient to show $B$ is a theorem. We also get reductio - if $A \vdash \neg A$, then $\neg A$. Arguments by cases of this sort are used very extensively in our paper, beginning in $\$ 1.3$ and repeating throughout.

\section{A note on Curry's paradox}

There is a question about whether our restricted quantifiers, which are added to $D K Q$, give rise to Curry's paradox. Now, one can use the logic $D K Q$ (without restricted quantifiers) for a full blown paraconsistent set theory, paradoxes and all, in which Curry sets $C=\{x: x \in x \rightarrow \perp\}$ can be generated. Brady has provided models showing that such a set theory is non-trivial. In this paper, set abstraction is used, so $x \in\{z: A(z)\}$ is synonymous with $A(x)$. But throughout, we are not concerned with sets larger than $\mathbb{R}$, nor do we do anything with sets per se, beyond very standard mathematical bookkeeping; and so naive set theory does not in fact seem to be required. Therefore worries about the contraction-like behavior of restricted quantifiers seems in part to be a moot point, because Curry sets will not arise. Syntactically, they just represent a (non-trivial) consequence relation $\vdash$.

Eventually, we do want paraconsistent real analysis to be compatible with paraconsistent set theory, including a purely paraconsistent construction of the 
reals. And then we will have to face concerns about validity curries [2]. The logic to use will be a substructural version of $D K Q$, which drops structural contraction,

$$
\frac{A, A \vdash B}{A \vdash B}
$$

as well as the axiom

$$
(A \rightarrow B) \wedge(A \rightarrow C) \rightarrow(A \rightarrow B \wedge C)
$$

We can assume the dual, argument by cases, in structural form:

$$
\frac{A \vdash C \quad B \vdash C}{A \vee B \vdash C}
$$

And thus all the 'validity Curries' that would afflict us in using $A \vdash B$ to define $\forall x \in A(x \in B)$, appear to be circumvented $\sqrt{16}$ Again, this is based on the very recent 2, which came to light after this paper was accepted for publication; for closely related issues about expressibility, see [12, ch. 26].

So our project is far from finished. Foundationalist system building aside, though, the present paper has simply aimed to demonstrate that consistencyreliant logic is not required to prove ordinary theorems in analysis. Rumors to the contrary notwithstanding, a paraconsistent logic can - and does - sustain long chains of ordinary mathematical reasoning.

\section{References}

[1] D. Batens, C. Mortensen, G. Priest, and J.-P. van Bendegem, editors. Frontiers of Paraconsistent Logic. Research Studies Press, Philadelphia, 2000.

[2] J. Beall and J. Murzi. Two flavors of curry paradox. Under review; see http://homepages.uconn.edu/ jcb02005/, 201x.

[3] R. Brady, editor. Relevant Logics and their Rivals, Volume II: A continuation of the work of Richard Sylvan, Robert Meyer, Val Plumwood and Ross Brady. Ashgate, 2003. With contributions by: Martin Bunder, Andre Fuhrmann, Andrea Loparic, Edwin Mares, Chris Mortensen, and Alasdair Urquhart.

\footnotetext{
${ }^{16}$ The loss of structural contraction is not so great as may be feared. When $A$ is a proven theorem, we may use $A$ as many times as we like. Most mathematically interesting arguments of the form $A \vdash B$ involve premises that are either axioms or theorems. Ergo the repeated use of these premises can be discharged all at once. So in practice unwieldy, irreducible statements like $A, \ldots, A \vdash B$ almost never come up. At worst it can be rephased as the weaker structural fact: $\frac{\vdash A}{\vdash B}$
} 
[4] R. Brady. Universal Logic. CSLI, Stanford, 2006.

[5] R. T. Brady. The non-triviality of dialectical set theory. In Priest et al. [23, pages $437-470$.

[6] D. Bridges and F. Richman. Varieties of Constructive Mathematics. Cambridge University Press, 1987.

[7] B. Brown and G. Priest. Chunk and permeate i: the infinitesimal calculus. Journal of Philosophical Logic, 33:379-88, 2004.

[8] A. B. Brunner and W. A. Carnielli. Anti-intuitionism and paraconsistency. Journal of Applied Logic, 3:161 - 184, 2005.

[9] S. Butchart. Binary quantifiers for relevant paraconsistent logic. To appear; presented at the Australasian Association for Philosophy Conference, Melbourne 2008.

[10] N. da Costa. Paraconsistent mathematics. In Batens et al. [1], pages 165180.

[11] N. C. A. da Costa. On the theory of inconsistent formal systems. Notre Dame Journal of Formal Logic, 15:497-510, 1974.

[12] H. Field. Saving Truth from Contradiction. Oxford University Press, 2008.

[13] N. D. Goodman. The logic of contradiction. Zeitschrift für Mathematische Logik und Grundlagen der Mathematik, 27(8 - 10):119-126, 1981.

[14] C. Lewis and C. Langford. Symbolic Logic. Dover, 1959.

[15] E. Mares. Relevant Logic. Cambridge University Press, Cambridge, UK, 2004.

[16] R. K. Meyer, R. Routley, and J. M. Dunn. Curry's paradox. Analysis, 39:124-128, 1978.

[17] C. Mortensen. Models for inconsistent and incomplete differential calculus. Notre Dame Journal of Formal Logic, 31(2):274 - 285, 1990.

[18] C. Mortensen. Inconsistent Mathematics. Kluwer Academic Publishers, Dordrecht; New York, 1995.

[19] C. Mortensen. Prospects for inconsistency. In D. Batens, C. Mortensen, G. Priest, and J.-P. van Bendegem, editors, Frontiers of Paraconsistent Logic, pages 203 - 208. Research Studies Press, Philadelphia, 2000. 
[20] C. Mortensen. Inconsistent mathematics: Some philosophical implications. In A. Irvine, editor, Handbook of the Philosophy of Science Volume 9: Philosophy of Mathematics. North Holland/Elsevier, 2009.

[21] G. Priest. In Contradiction: A Study of the Transconsistent. Oxford University Press, Oxford, 2006. Second edition.

[22] G. Priest. An Introduction to Non-Classical Logic. Cambridge, 2008. Second Edition.

[23] G. Priest, R. Routley, and J. Norman, editors. Paraconsistent Logic: Essays on the Inconsistent. Philosophia Verlag, Munich, 1989.

[24] R. Routley. Ultralogic as universal? Relevance Logic Newsletter, 2:51-89, 1977. Reprinted in [25].

[25] R. Routley. Exploring Meinong's Jungle and Beyond. Philosophy Department, RSSS, Australian National University, Canberra, 1980. Departmental Monograph number 3.

[26] R. Routley, V. Plumwood, R. K. Meyer, and R. T. Brady. Relevant Logics and their Rivals. Ridgeview, 1982.

[27] Z. Weber. Transfinite numbers in paraconsistent set theory. Review of Symbolic Logic, 3(1):71 - 92, 2010. 


\section{University Library}

\section{- M M I N E R VA A gateway to Melbourne's research publications}

Minerva Access is the Institutional Repository of The University of Melbourne

Author/s:

McKubre-Jordens, M;Weber, Z

Title:

Real Analysis in Paraconsistent Logic

Date:

2012-10-01

Citation:

McKubre-Jordens, M. \& Weber, Z. (2012). Real Analysis in Paraconsistent Logic. Journal of Philosophical Logic, 41 (5), pp.901-922. https://doi.org/10.1007/s10992-011-9210-6.

Persistent Link:

http://hdl.handle.net/11343/282773 